\title{
eJRIEPS
}

Ejournal de la recherche sur l'intervention en éducation physique et sport

$44 \mid 2019$

Varia

\section{Soutenance d'HDR}

\section{OpenEdition \\ Journals}

Édition électronique

URL : http://journals.openedition.org/ejrieps/447

DOI : $10.4000 /$ ejrieps.447

ISSN : 2105-0821

Éditeur

ELLIADD

Référence électronique

"Soutenance d'HDR », eJRIEPS [En ligne], 44 | 2019, mis en ligne le 01 janvier 2019, consulté le 24 septembre 2020. URL : http://journals.openedition.org/ejrieps/447 ; DOI : https://doi.org/10.4000/ ejrieps. 447

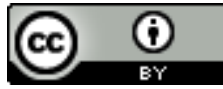

La revue eJRIEPS est mise à disposition selon les termes de la Creative Commons Attribution 4.0 International License. 


\section{Actualités de la recherche en intervention}

\section{Recension}

Marc Deleplace, Daniel Bouthier, Pierre Villepreux (dir.). (2018). René Deleplace. Du rugby de mouvement à un projet global pour l'EPS et les STAPS. Villeneuve d'Ascq : Presses universitaires du Septentrion.

Recension

Didier Barthès

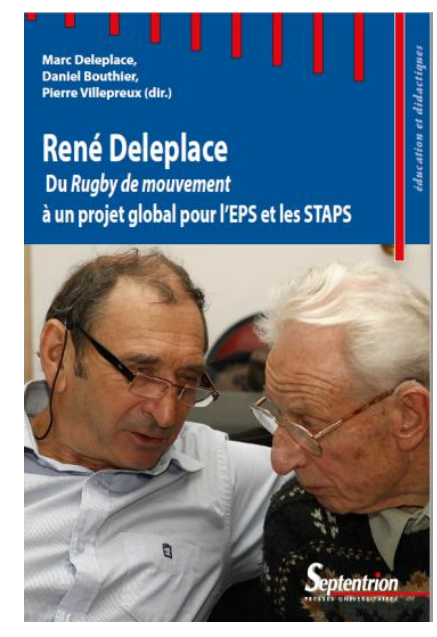

L'ouvrage de 270 pages se présente sous une forme contributive de 7 auteurs principaux pour 22 contributeurs, sous la direction conjointe de Marc Deleplace, Daniel Bouthier et Pierre Villepreux.

II a l'ambition de regrouper un ensemble de contributions à propos et autour de l'œuvre et l'engagement militant de René Deleplace, de ses conceptions et théories du rugby mais également à propos de la formation en éducation physique et en rugby fédéral comme scolaire, voire de son expérience concernant le cor d'harmonie. Les différentes contributions sont enrichies par une suite de textes publiés ou non par René Deleplace; textes présentés et analysés par les différents auteurs principaux. L'ensemble de ces contributions constitue sans conteste, le témoignage 


\section{eJRIEPS 44 janvier 2019}

d'une expérience humaine "à hauteur d'homme ", une expérience en lien avec l'ensemble des facettes constituant l'œuvre de René Deleplace. Cette expérience qui va du sport en général, au rugby bien évidemment, mais encore à l'éducation physique et sportive et la formation en STAPS, relie cette dimension sportive au domaine de la musique et plus particulièrement au cor d'harmonie. C'est un projet global qui nous est proposé, tant pour l'EPS et les STAPS que pour l'entrainement et la réflexion épistémologique et axiologique liée intimement avec l'expérience de terrain, celle de l'entraineur, du militant et du formateur. Car la particularité de l'œuvre présentée tient surtout au souci d'articuler les problématiques de terrain, celles de la formation et de l'entrainement, à des questions et des enjeux théoriques, épistémiques voire politiques; en cela, cet ouvrage rend compte d'une dimension émancipatrice de l'activité motrice humaine telle qu'a pu la mettre en œuvre René Deleplace.

L'ouvrage se présente en 3 sections conséquentes en volume et en types de contributions, sections dans lesquelles différentes thématiques sont présentées en plusieurs chapitres et contributeurs. Une bibliographie sélective par auteur est proposée en fin d'ouvrage. Elle vient en complément des références de bas de page dans chacune des contributions.

L'idée de proposer un ensemble de réflexions, témoignages et analyses, est tout à fait intéressant dans la mesure où ce type de travail n'a pas été encore réalisé, c'est là l'intérêt principal de cet ouvrage que de replacer dans la diachronie, les différentes étapes de l'œuvre de René Deleplace, de son cheminement conceptuel dans les différents domaines de son activité professionnelle, militante comme sportive. De cette façon, les éléments contextuels, ceux du terrain et de la formation, viennent éclairer de façon tout à fait originale et pertinente la démarche de René Deleplace tout en fournissant ainsi l'étayage et la cohérence théorique et méthodologique de son œuvre.

Ainsi, articuler le rugby, la formation physique et le cor d'harmonie constitue un exercice délicat qui est en définitive plutôt bien mené et pertinent. On saisit bien que l'objectif de l'ouvrage ne soit pas de faire un panégyrique de René Deleplace et de son œuvre mais bien de présenter de façon exhaustive ses idées et discuter ses conceptions concernant le rugby et de manière plus générale la formation physique 


\section{eJRIEPS 44 janvier 2019}

et culturelle de l'homme sportif. II s'agit là d'une conception humaniste résolument ancrée dans son temps.

La préface pose le cadre général des contributions qui vont suivre. Elle présente de manière pertinente les différentes dimensions de l'œuvre de René Deleplace tout en essayant de circonscrire l'espace du " chantier deleplacien " dans ses dimensions anthropologiques. Ainsi, les contributions prendront un caractère souvent dynamique et actuel sous la forme de témoignages d'expérience personnelle et de pratiques d'interventions professionnelles éclairées par une dimension théorique de bon niveau.

Après une biographie fidèle menée par Marc Deleplace puis par Jacques Giraud, la section 1, coordonnée par Marc Deleplace et Daniel Bouthier, est consacrée au cadre théorique développé par René Deleplace. L'objectif est de définir un objet scientifique selon une trajectoire définie par le passage de la pratique sociale de référence, le rugby, à une pratique scolaire en termes d'activité physique et sportive. Cette section rend compte du cheminement d'une modélisation de l'activité physique s'appuyant sur une démarche empirique, celle de l'expérience de joueur, d'entraineur et de formateur favorisant l'émergence d'une réelle épistémologie des activités physiques, celle-ci ne pouvant s'appuyer que sur la réalité de l'expérience.

La deuxième section coordonnée par Marc Deleplace et Serge Reitchess, aborde la dimension culturelle et sociale de l'œuvre de René Deleplace. Cette section éclaire le caractère militant des convictions qui ont organisé et justifié l'activité de René Deleplace. Elle articule l'action professionnelle comme syndicale et sportive, en lui donnant une certaine unité de conviction. La dimension didactique liée intimement à la réflexion épistémologique permet aux auteurs de souligner la recherche de cohérence chez René Deleplace. Celle-ci ne peut s'envisager que dans une approche systémique articulant le geste technique et le contexte dans lequel celui-ci s'accomplit.

La troisième section coordonnée par Marc Deleplace propose, après une partie introductive, une anthologie de textes publiés ou non, classés chronologiquement. Des textes choisis, présentés et analysés par Marc Deleplace, Serge Reitchess, Daniel Bouthier ou encore Jacques Dury, sont organisés selon 4 thématiques: théorie du rugby, formation en APS comme champ scientifique, le lien avec l'EPS et enfin celui plus général des apprentissages moteurs dont ceux en lien avec le cor 


\section{eJRIEPS 44 janvier 2019}

d'harmonie. Cette section est intéressante du point de vue historique et épistémologique car elle fournit un matériau pertinent de réflexion pour le lecteur. Par ailleurs les éléments d'analyse fournis par les auteurs, viennent éclairer de façon tout à fait intéressante les différentes dimensions de l'œuvre de René Deleplace.

Didier Barthes

ESPE Midi-Pyrénées - Université Toulouse Jean-Jaurès.

Si tu as besoin d'un laboratoire de référence :

UMR - EFTS - UT2J

\section{Soutenance d'HDR}

Mathilde Musard, maître de conférences à l'université de Franche-Comté a soutenu son Habilitation à Diriger les Recherches (HDR), intitulée " La dynamique curriculaire en Education Physique et Sportive. Vers une approche comparatiste en didactique » le jeudi 25 Octobre 2018 à Besançon.

\section{Le jury était composé de :}

C. AMADE-ESCOT, Professeure émérite, Université de Toulouse (Présidente)

J. BISAULT, Professeur, Université d'Amiens (Rapporteur)

S. BRAU-ANTONY, Professeur, Université de Reims (Garant scientifique)

M. COQUIDE, Professeure émérite, École Normale Supérieure de Lyon J-F. DESBIENS, Professeur des Universités, Université de Sherbrooke (Rapporteur) M. LOQUET, Professeure, Université de Rennes (Rapporteure)

D. PASCO, Professeur, Université de Besançon (Garant institutionnel)

\section{Résumé}

Les recherches sur les curriculums sont cloisonnées : elles portent plus souvent sur les curriculums prescrit et potentiel (Martinand, 2014) à partir de données textuelles (textes officiels, analyse de manuels pédagogiques, documents, entretiens...) et moins sur le curriculum en actes tel qu'il se co-construit en classe dans les interactions entre l'enseignant et les élèves (Jonnaert, 2011; Lenzen, 2012 ; Audigier \& Tutiaux-Guillon, 2008). 


\section{eJRIEPS 44 janvier 2019}

La problématique de cette habilitation à diriger les recherches s'inscrit dans le dialogue entre les traditions curriculaire et didactique (Gundem \& Hopmann, 1998 ; Audigier, Crahay \& Dolz, 2006 ; Lebeaume, 2011 ; Martinand, 2014) et vise à appréhender la complexité de la dynamique curriculaire en EPS au regard des contenus, qu'ils soient prescrits, interprétés ou co-construits par l'enseignant et les élèves in situ. II s'agit de mener des enquêtes à différentes échelles, depuis le curriculum prescrit jusqu'au curriculum en actes, en fonction de différentes temporalités (années, séquence, leçon...) et en lien avec les composantes du curriculum (visées éducatives, contenus, progression, évaluation, etc.).

Les recherches empiriques sont revisitées à partir d'un cadre de didactique curriculaire selon trois axes : l'étude des curriculums prescrit et potentiel ; l'étude du curriculum en actes et l'étude de la dynamique curriculaire.

Le programme de recherche discute des aspects théoriques et méthodologiques en vue d'élaborer un modèle pour l'étude de la dynamique curriculaire. II s'enrichit à travers l'ancrage dans les approches comparatistes en didactique au sein des sciences de l'homme et de la société et l'ouverture sur différentes visées de recherche pour contribuer à la (trans)formation des enseignants.

\section{Soutenance de thèse}

Marcos Roberto Godoi a présenté sa thèse en décembre 2017 à la Faculté des sciences de l'éducation, Université de Montréal, en vue de l'obtention du grade de Philosophiae Doctor (Ph.D.) en sciences de l'éducation, option psychopédagogie. Cette thèse est intitulée "Le "travail curriculaire" des enseignants en éducation physique : du travail prescrit au travail réel » est disponible dans Papyrus de l'UdeM via http://hdl.handle.net/1866/19975

\section{Le jury était composé de :}

Francisco Loyola, président

Cecilia Borges, directrice de recherche

François Vandercleyen, membre

Yannick Lémonie, examinateur externe

Serge Larivée, représentant de la doyenne 


\section{eJRIEPS 44 janvier 2019}

\section{Résumé}

Dans cette recherche de doctorat, nous analysons le « travail curriculaire » des enseignants en éducation physique. Le travail curriculaire est l'ensemble des processus d'interprétation, d'adaptation et de transformation du curriculum prescrit par les enseignants pour le rendre enseignable aux élèves (Tardif et Lessard, 1999). En 2012, il y a eu la mise en œuvre du curriculum municipal d'éducation physique (CMÉP) pour les écoles municipales de Cuiabá (Moreira, 2012), dans l'État du Mato Grosso, au Brésil. Cependant, on en savait très peu sur comment les enseignants l'intègrent à leurs enseignements en salle de classe. En ce sens, notre question de recherche est : comment les enseignants interprètent-ils et transforment-ils le curriculum prescrit en salle de classe? Autrement dit, comment réalisent-ils le travail curriculaire? Concernant notre cadre théorique, il s'appuie sur la sociologie du travail enseignant et sur l'ergonomie française, plus particulièrement sur l'approche de la clinique de l'activité. Notre recherche est mixte, mais avec un accent plus fort placé sur la perspective qualitative. En outre, l'étude peut être classifiée comme une recherche-intervention selon l'approche historico-développementale. Dans la première phase, nous avons administré un questionnaire à 73 enseignants pour connaître leurs perceptions à l'égard du CMÉP et du programme de formation continue qui a été offert pour appuyer l'implantation du curriculum. Dans la deuxième phase, nous avons mené une étude multi-cas avec quatre enseignants, trois femmes et un homme, dont deux étaient novices (E1 et E2) et deux étaient expérimentés (E3 et E4). Nous avons consulté des documents (le curriculum prescrit, les projets pédagogiques des écoles, les planifications des enseignants) et nous avons fait des entrevues semi- dirigées au début de la recherche et à la fin de l'année scolaire 2015. De plus, nous avons observé et tourné des vidéos de leurs leçons pendant une période de deux mois. Ensuite, nous avons appliqué la méthode de l'autoconfrontation simple et croisée (Clot, 2008), où nous avons pu accéder aux points de vue sur les activités en salle de classe des enseignants participants à l'étude multi-cas. Parmi les principaux résultats de notre recherche, nous pouvons souligner que : concernant la perception générale positive du CMÉP, 82,19\% des enseignants trouvent qu'il est avantageux d'enseigner conformément au CMÉP. Par rapport à l'usage du CMÉP, presque $80 \%$ des enseignants utilisent toujours ou presque toujours ce curriculum pour faire leurs planifications annuelles, $76,72 \%$ en 


\section{eJRIEPS 44 janvier 2019}

font l'usage pour planifier leurs séances, et $72,61 \%$ l'utilisent en l'adaptant. En ce qui a trait aux aspects qui entravent l'implantation du CMÉP, le manque de matériel iv pédagogique $(41,1 \%)$ et les installations inadéquates $(34,25 \%)$ sont les facteurs qui empêchent le plus la mise en œuvre du curriculum. Par rapport à la formation continue, $74 \%$ des enseignants estiment qu'elle offre l'appui suffisant pour l'implantation du CMÉP et qu'elle a été fonctionnelle pour presque $90 \%$ des enseignants. Par rapport aux résultats de l'étude multi-cas, nous soulignons que : les enseignants utilisent le CMÉP pour faire leurs planifications annuelles, à moyen ou court termes, mais ils l'interprètent, l'adaptent et le transforment lors de l'enseignement en classe avec leurs élèves, chacun à sa façon et selon leurs connaissances de la matière, du curriculum et des élèves, des ressources et des espaces disponibles, mais en les transformant et en les adaptant ceux- ci aussi. En bref, les facteurs contextuels et personnels du travail curriculaire influent sur cette adaptation du curriculum. De plus, notre étude a montré que les enseignants sont des interprètes, acteurs, auteurs et constructeurs du curriculum d'éducation physique enseigné en classe et que les élèves sont co-auteurs des leçons avec leurs enseignants. 